\title{
CARBON MONOXIDE IN THE MAGELLANIC CLOUDS
}

\author{
F.P. ISRAEL \\ Sterrewacht Leiden \\ PO Box 9513 \\ 2300 RA Leiden \\ The Netherlands \\ Th. de GRAAUW \\ Laboratory for Space Research \\ PO Box 800 \\ 9700 AV Groningen \\ The Netherlands
}

\section{Introduction}

Molecular gas is a major constituent of the interstellar medium of all late-type galaxies. Virtually all of it is in the form of cold molecular hydrogen $\left(\mathrm{H}_{2}\right)$ which today cannot be observed directly. However, the tracer molecule carbon monoxide (CO) (relative abundance $10^{-5}$ ) is easily detected. For the Magellanic Clouds (MCs), CO studies are of specific importance. The Clouds are rich in $\mathrm{HI}$, and if we can establish the presence of significant amounts of $\mathrm{H}_{2}$ as well, this will influence our estimates of the global rate of star formation and its history. Complexes of presently quiescent molecular gas may betray regions primed for star formation, but not yet in action. Detailed studies of the $\mathrm{HI}, \mathrm{HII}$ and $\mathrm{H}_{2}$ and young star content may provide estimates of star formation efficiences on scales of a kiloparsec.

There is another important aspect. At present a controversy rages on how to estimate $\mathrm{H}_{2}$ amounts from observations of the optically thick molecule $\mathrm{CO}$. At the core of this controversy lies the question: to what extent does the $\mathrm{CO}$ emission from $\mathrm{H}_{2}$ clouds reflect variations in dust content, metallicity and the ambient UV radiation field (cf. Israel 1988). Here, the MCs are important because of their physical condition: metallicity lower and radiation field somewhat higher than in the Galaxy. They thus provide a good laboratory in which to test environmental effects.

The MCs present both a global and a detailed view. Today, millimetre telescopes offer linear resolutions better than about $15 \mathrm{pc}$. This is much higher than presently obtainable far-infrared (FIR) or HI resolutions.

\section{History}

Before 1980, little was known about $\mathrm{CO}$ or $\mathrm{H}_{2}$ in the MCs (cf. Israel 1984). The first $\mathrm{CO}$ detection in the LMC (Huggins et al. 1975) was followed by a limited CO(2-1) survey (Israel et al. 1982, 1986). Although only a minute fraction of the LMC and SMC was sampled, it was clear that in the Clouds, especially the SMC, CO emission is weak compared to the Galaxy. This is 
explained by $\mathrm{CO}$ photodissociation due to the strong radiation field, low dust content and low metallicity of the Clouds. The weakness of $\mathrm{CO}$ does not imply small amounts of $\mathrm{H}_{2}$; molecular hydrogen is only weakly affected by photodissociation in the Clouds.

The first complete CO map of the LMC, produced by Cohen et al. (1988) with a linear resolution of $200 \mathrm{pc}$, shows CO clouds near major star formation regions such as N11. The Bar can be recognized but 30 Doradus is weak in CO. In contrast, the region south of 30 Doradus, notably near N159, shows strong CO emission extending southwards from the HII regions to an optically inconspicuous area. This may be the major star formation region of the future. The $\mathrm{CO}$ map is very similar to thw warm-dust map published by Schwering (1988) but differs from the HI map (Rohlfs et al. 1984). Cohen et al. (1988) conclude that for a given amount of $\mathrm{H}_{2}, \mathrm{CO}$ emission in the LMC is about six times weaker than in the Galaxy. Thus, use of the Galactic conversion leads to an underestimation of $\mathrm{H}_{2}$, as also concluded by Israel $e t$ al. (1986).

\section{The SEST Key Project}

Recently, the Swedish-ESO millimetre telescope SEST has become available at ESO. With its 40" beam, mapping the MCs is time consuming. For this reason, a consortium of ESO and Swedish observers was formed. ESO and Sweden have rewarded the consortium with Key Project status, guaranteeing a continuation of the study of $\mathrm{CO}$ in the Clouds. In the following, we present preliminary results from the initial Swedish work and the work done until now by the consortium. So far, several molecular clouds associated with the Doradus HII regions, including 30 Doradus itself, have been mapped in the LMC, as well as a small cloud near N55, and a large complex associated with N11.

We have also obtained a several-degrees-long stripmap at constant R.A. through the Doradus region and have started mapping clouds found in this strip. A similar degrees-long strip through Doradus and the Bar, at constant Dec, is now in progress. In the SMC, we have mapped a few isolated small clouds, notably N88, and a map of the relatively bright southwestern part of the Bar is in progress. We have also conducted a survey of selected positions in the Clouds. The presence of IRAS infrared peaks gives a much better chance of detecting $\mathrm{CO}$ than the presence of dark clouds or HII regions per se. Thus, in the LMC survey (which took place after the SMC survey), we concentrated on the IRAS peaks given by Schwering and Israel (1990). We also measured ${ }^{13} \mathrm{CO}$ strengths on the brightest ${ }^{12} \mathrm{CO}$ peaks. The survey yielded, in the LMC, $75{ }^{12} \mathrm{CO}$ detections, 10 upper limits and $36{ }^{13} \mathrm{CO}$ measurements and, in the SMC, $18{ }^{12} \mathrm{CO}$ detections, 17 upper limits and $6^{13} \mathrm{CO}$ measurements.

\section{Results of the preliminary $\mathrm{CO}$ survey}

Although IRAS sources give a good chance of detecting CO, the CO/FIR ratio has a large scatter. Much of this is caused by the different IRAS and SEST beam sizes, but at least part of it is intrinsic. It appears that the integrated $\mathrm{CO}$ emission is better correlated with dust temperature than with dust column density. $\mathrm{CO}$ emission peaks at dust temperatures between 35 and $40 \mathrm{~K}$. At higher temperatures emission drops, presumably because photodissociation starts dominating.

We confirm the remarkable weakness of $\mathrm{CO}$ in the Clouds. Our survey is biased towards the strongest $\mathrm{CO}$ sources in the Clouds. Yet, we find a mean detected integrated $\mathrm{CO}$ strength of only $11 \mathrm{~K} \mathrm{~km} \mathrm{~s}^{-1}$ in the LMC and $3 \mathrm{~K} \mathrm{~km} \mathrm{~s}^{-1}$ in the SMC. Comparison with 'average' Galactic positions observed with the same linear beamsize yields a mean of $30 \mathrm{~K} \mathrm{~km} \mathrm{~s}^{-1}$ for the latter. Only seven (10\%) LMC detections are at this brightness level; none in the SMC. Thus, CO in the LMC 
is at least three times weaker than Galactic $\mathrm{CO}$, whereas $\mathrm{CO}$ in the SMC is at least ten times weaker!

In the Clouds, the ${ }^{12} \mathrm{CO} /{ }^{13} \mathrm{CO}$ isotopic ratio is about 10 for the peaks and 14 for integrated profiles, as compared to peak ratios between 5 and 8 in the Galaxy. In the LMC, peak ratios range from 7 to 14. Statistics for the SMC are still too poor for a conclusion. We have calculated $\mathrm{H}_{2}$ column densities both from integrated ${ }^{12} \mathrm{CO}$ intensities and from ${ }^{12} \mathrm{CO}$ and ${ }^{13} \mathrm{CO}$ measurements. Surprisingly, the two $\mathrm{H}_{2}$ column density sets are not linearly related. Rather, ${ }^{13} \mathrm{CO}$-inferred $\mathrm{H}_{2}$ column densities increase only very slowly with ${ }^{12} \mathrm{CO}$-inferred $\mathrm{H}_{2}$ column densities. Thus, meaningful $\mathrm{H}_{2}$ column densities and masses can only be obtained from modelling; use of the Galactic conversion leads to false results.

\section{Maps of giant molecular cloud complexes}

So far, only a small fraction of the Magellanic Clouds has been mapped. Here, we briefly discuss three maps. A preliminary map of the Doradus HII regions was made by the Swedish observers during commissioning time. They found six major $\mathrm{CO}$ clouds and a dozen lesser ones, and used Local Velocity Gradient models to conclude that the $\mathrm{J}=1-0{ }^{12} \mathrm{CO}$ has small optical depths around unity in these clouds, that the fractional abundance of ${ }^{12} \mathrm{CO}$ is only one tenth of that in the Galaxy, and that for a given amount of $\mathrm{H}_{2}, \mathrm{CO}$ emission from these clouds may be up to six times weaker than from Galactic clouds. The latter conclusion confirms the results obtained by Israel et al. (1986), Cohen et al. (1988) and also the result mentioned in section 4.

The consortium has mapped most of the HII region complex N11 (Fig. 1). In a region of about $500 \times 500 \mathrm{pc}$, we find 21 well-separated CO clouds, of typical size $20-30 \mathrm{pc}$. The structure of the N11 complex is similar to that of the $200 \mathrm{pc}$ diameter Orion molecular complex. Between bright filaments and clumps, the Orion map shows weaker emission not seen in N11, probably because of sensitivity limitations. The clouds follow the IRAS infrared map closely, especially at 25 and $60 \mu \mathrm{m}$. In the southwest, $\mathrm{CO}$ clouds form a 'ring' around a rich cluster of luminous stars; apparently all the $\mathrm{CO}$ there has been consumed, destroyed or driven out.

Mapping of the weak CO clouds in the southwest bar of the SMC is still in progress. Several clouds at different radial velocities have been found near major IRAS sources (Fig. 2). The southwest Bar is a confused area because here the line of sight through the SMC is kiloparsecs long. Indeed, individual clouds overlap in integrated CO maps. They must be carefully separated in position-velocity space before, for example, virial masses can be obtained.

\section{Molecular cloud masses}

For about 50 LMC clouds, mostly in two complexes (Doradus and N11) we can compare the virial and the $\mathrm{CO}$ mass derived in the usual manner. In principle both should be independent of actual $\mathrm{CO}$ abundance. We find that in the (virial) mass range of $2 \times 10^{3} \mathrm{M}_{\mathrm{e}}$ to $2 \times 10^{5} \mathrm{M}_{\theta}$, virial mass is higher by a factor of 1.5 to 6 with a mean of 3 times higher. Masses derived from both ${ }^{12} \mathrm{CO}$ and ${ }^{13} \mathrm{CO}$ are lower than the virial mass by a factor of 5 to 10 , although this is not independent from abundances. Moreover, the ratio between the derived masses depends on (virial) mass. This is the same effect already mentioned in section 4. Although a final conclusion awaits proper modelling, taking into account the physical environment, it appears that $\mathrm{H}_{2}$ masses and column densities in the LMC are comparable to those of Galactic molecular clouds, whereas the CO-to- $\mathrm{H}_{2}$ conversion 


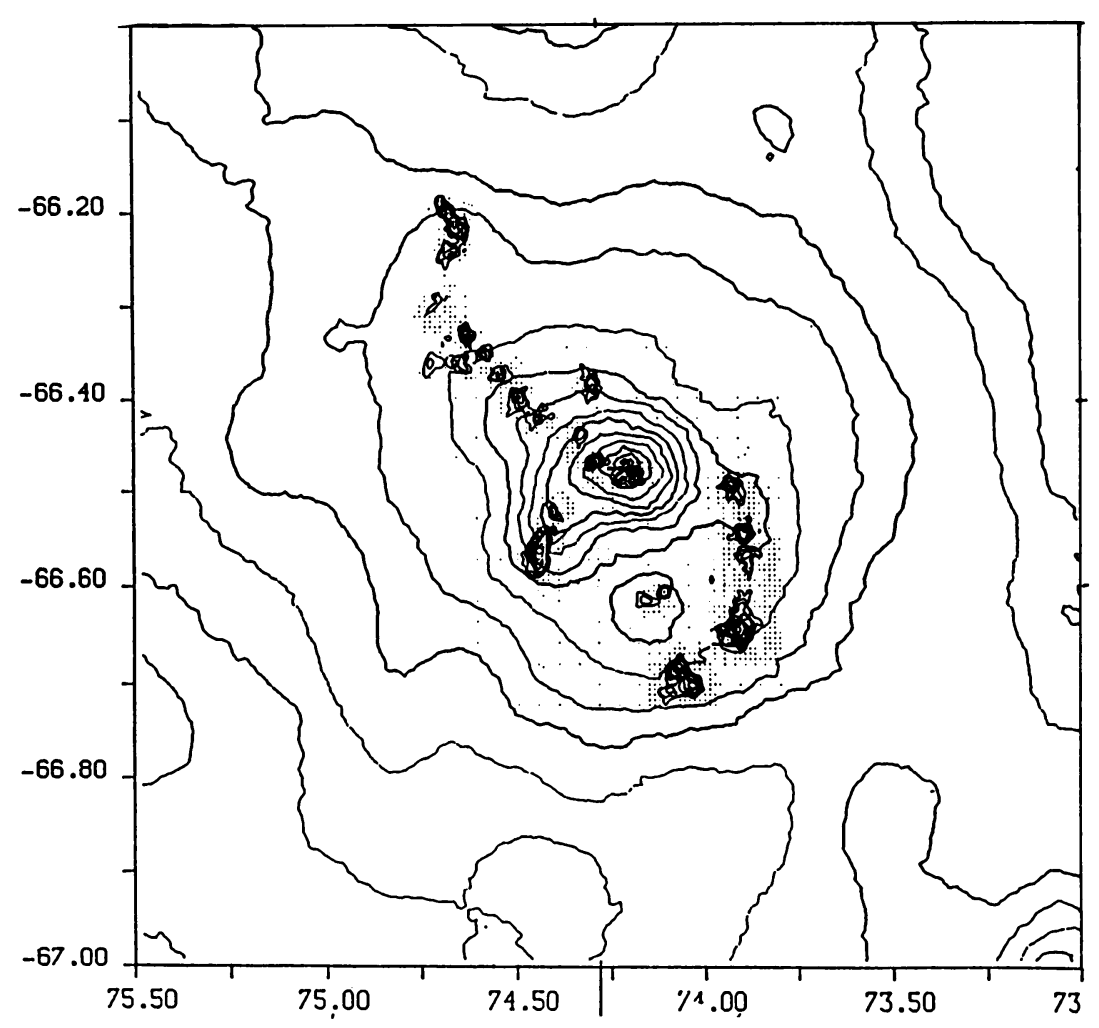

Figure 1. Map of CO clouds in N11 superposed on GEISHA $100 \mu \mathrm{m}$ IRAS map. Distribution of CO clouds follows the (lower resolution) infrared contours. Positions sampled for $\mathrm{CO}$ are indicated by dots; 1950 coordinates are given in degrees.

factor is about three times higher than in the Galaxy. As the virial mass was estimated conservatively, this factor is more likely to be higher than lower. This result can be plausibly ascribed to relatively important photodissociation in the LMC (cf. Israel et al. 1986). To resolve this issue, modelling of the sort discussed by Maloney (1990) is an urgent necessity.

\section{Summing up}

The results obtained confirm that the $\mathrm{CO}$ emission from the Clouds is weaker than that from the Galaxy by a significant factor, for given amounts of $\mathrm{H}_{2}$. At least the LMC contains almost certainly 'normal' amounts of $\mathrm{H}_{2}$. Molecular complexes in the LMC and SMC consist mostly of 20-30 pc-sized clouds, although observations at higher resolutions probably will show important substructure in these clouds. Many questions still need to be answered. What will modelling tell us about the quantitative influence of environmental conditions on LMC molecular cloud properties? Do cold CO clouds not engaged in star formation ('Taurus dark clouds') exist? What is the condition of molecular clouds in the extreme environment of the SMC? What can we conclude on the interaction between molecular clouds and star formation? 


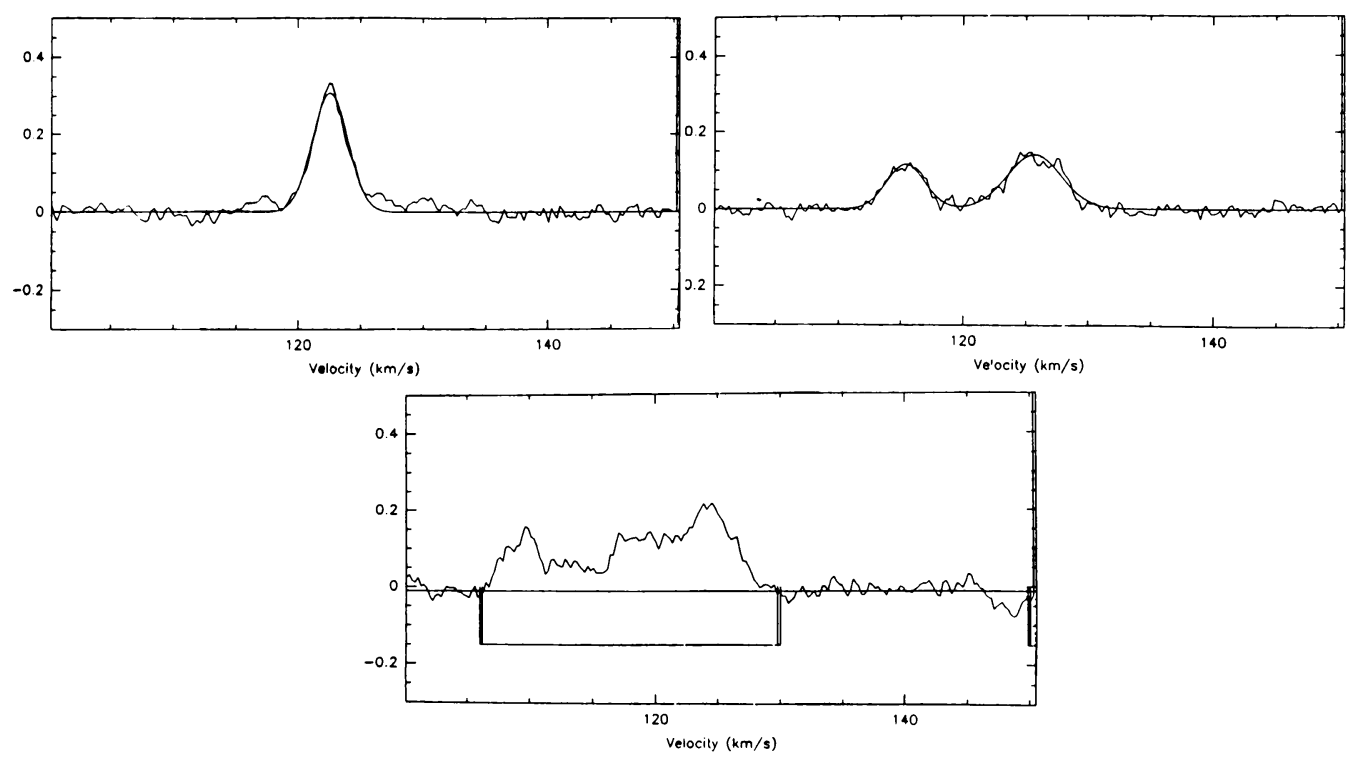

Figure 2. CO profiles of cloud peaks in the SMC southwest Bar. Individual clouds are well defined in top profiles, but bottom profile shows at least three confused components. $\mathrm{CO}$ intensities are in $\mathrm{K}$, not corrected for main-beam efficiency.

\section{References}

Cohen, R.S., Dame, T.M., Garay, G., Montani, J., Rubio, M., Thaddeus, P. (1988), Ap. J. 331, L95.

Huggins, P.J., Gillespie, A.R., Phillips, T.G., Gardner, F., Knowles, S. (1975), M.N.R.A.S. 173, 69P.

Israel, F.P. (1984), IAU Symp 108 Structure and Evolution of the Magellanic Clouds, S. van den Bergh, K.S. de Boer (eds.) (Reidel: Dordrecht) p.139.

Israel, F.P. (1988), Millimetre and Submillimetre Astronomy, R.D.Wolstencroft, W.B. Burton (eds.) (Kluwer: Dordrecht) p.281.

Israel, F.P., de Graauw, Th., Lidholm, S., van de Stadt, H., de Vries, C. (1982), Ap. J. 262, 100.

Israel, F.P., de Graauw, Th., van de Stadt, H., de Vries, C.P. (1986), Ap. J. 303, 186.

Maloney, P. (1990), The Interstellar Medium in Galaxies, H.L. Thronson, J.M. Shull (eds.) (Kluwer: Dordrecht) p.493.

Rohlfs, K., Kreitschmann, J., Siegman, B., Feitzinger, J.V. (1984), Astr. Ap. 137, 343.

Schwering, P.B.W. (1988), PhD. Thesis, Leiden University (NL).

Schwering, P.B.W., Israel, F.P. (1990), Atlas and Catalogue of Infrared Sources in the Magellanic Clouds (Kluwer: Dordrecht). 